\title{
Fuzzy Logic-Based Histogram Equalization for Image Contrast Enhancement
}

\author{
V. Magudeeswaran ${ }^{1}$ and C. G. Ravichandran ${ }^{2}$ \\ ${ }^{1}$ Department of Electronics and Communication Engineering, P.S.N.A College of Engineering and Technology, Dindigul, \\ Tamil Nadu 624622, India \\ ${ }^{2}$ Ratnavel Subramaniam (RVS) College of Engineering and Technology, Dindigul, Tamil Nadu 624005, India
}

Correspondence should be addressed to V. Magudeeswaran; magudeeswaran@gmail.com

Received 23 May 2013; Revised 27 June 2013; Accepted 27 June 2013

Academic Editor: Erik Cuevas

Copyright (C) 2013 V. Magudeeswaran and C. G. Ravichandran. This is an open access article distributed under the Creative Commons Attribution License, which permits unrestricted use, distribution, and reproduction in any medium, provided the original work is properly cited.

Fuzzy logic-based histogram equalization (FHE) is proposed for image contrast enhancement. The FHE consists of two stages. First, fuzzy histogram is computed based on fuzzy set theory to handle the inexactness of gray level values in a better way compared to classical crisp histograms. In the second stage, the fuzzy histogram is divided into two subhistograms based on the median value of the original image and then equalizes them independently to preserve image brightness. The qualitative and quantitative analyses of proposed FHE algorithm are evaluated using two well-known parameters like average information contents (AIC) and natural image quality evaluator (NIQE) index for various images. From the qualitative and quantitative measures, it is interesting to see that this proposed method provides optimum results by giving better contrast enhancement and preserving the local information of the original image. Experimental result shows that the proposed method can effectively and significantly eliminate washed-out appearance and adverse artifacts induced by several existing methods. The proposed method has been tested using several images and gives better visual quality as compared to the conventional methods.

\section{Introduction}

The main objective of an image enhancement is to bring out the hidden image details or to increase the image contrast with a new dynamic range. Histogram equalization (HE) is one of the most popular techniques used for image contrast enhancement, since HE is computationally fast and simple to implement $[1,2]$. HE performs its operation by remapping the gray levels of the image based on the probability distribution of the input gray levels. However, HE is rarely employed in consumer electronic applications such as video surveillance, digital camera, and television, since HE tends to introduce some annoying artifacts and unnatural enhancement, including intensity saturation effect [3]. One of the reasons for this problem is that HE normally changes the brightness of the image significantly and thus makes the output image, become saturated with very bright or dark intensity values.
Hence, brightness preserving is an important characteristic that needs to be considered in order to enhance the image for consumer electronic products.

In order to overcome the limitations of $\mathrm{HE}$ and to preserve image brightness, several brightness preserving histogram equalization techniques have been proposed. At first, Kim proposed brightness preserving bi-Histogram equalization (BBHE) [4], BBHE divides the input image histogram into two parts based on the mean of the input image, and then each part is equalized independently. Consequently, the mean brightness is preserved because the original mean brightness is retained. Wan et al. introduced dualistic subimage histogram equalization (DSIHE), which is similar to BBHE except that the median of the input image is used for histogram partition instead of mean brightness [5]. Chen and Ramli proposed minimum mean brightness error bihistogram equalization (MMBEBHE), which is the extension 
of $\mathrm{BBHE}$ method that provides maximal brightness preservation [6]. This algorithm finds the minimum mean brightness error between the original and the enhanced image. It employs the optimal point as the separating point instead of the mean or median of the input image. Though these methods can perform good contrast enhancement, they also cause more annoying side effects depending on the variation of gray level distribution in the histogram. Recursive mean separate HE (RMSHE) is another improved version of $\mathrm{BBHE}$ [7]. This method is recursively separates the histogram into multi-subhistograms instead of two subhistograms as in the $\mathrm{BBHE}$. Initially, two subhistograms are created based on the mean brightness of the original histogram. Subsequently, the mean brightness from the two subhistograms obtained earlier are used as the second and third separating points in creating more sub-histograms. In a similar fashion, the algorithm is executed recursively until the desired numbers of sub-histograms are met. Then, the HE approach is applied independently on each of the subhistograms. The methods discussed previously are based on dividing the original histogram into several sub-histograms by using either the median or mean brightness. Although the mean brightness is well preserved by the aforementioned methods, these methods cannot further expand the region of subhistogram that is located near to the minimum or maximum value of the dynamic range. However, it is also not free from side effects such as washed-out appearance, undesirable checkerboard effects, and significant change in image brightness.

In order to deal with the problem mentioned above, Abdullah-Al-Wadud et al. introduced a dynamic histogram equalization (DHE) technique [8]. DHE partitions the original histogram based on local minima. However, DHE does not consider the preserving of brightness. For this purpose, Ibrahim and Kong proposed brightness preserving dynamic histogram equalization (BPDHE) [9]. This method partitions the image histogram based on the local maxima of the smoothed histogram. It assigns a new dynamic range to each partition. Finally the output intensity is normalized to make the mean intensity of the resulting image equal to the input one. Although, the BPDHE performs well in mean brightness preserving, the ratio for brightness normalization plays an important role. A small ratio value leads to insignificant contrast enhancement. For large ratio (i.e., ratio value more than 1), the final intensity value may exceed the maximum intensity value of the output dynamic range. The exceed pixels will be quantized to the maximum intensity value of gray levels and produce intensity saturation problem (in MATLAB environment). Brightness preserving dynamic fuzzy histogram equalization (BPDFHE) has been proposed by Sheet et al. which is an enhanced version of BPDHE [10]. The BPDFHE technique manipulates the image histogram in such a way that no remapping of the histogram peaks takes place, while only redistribution of the gray-level values in the valley portions between two consecutive peaks takes place. The results using BPDFHE method show well-enhanced contrast and little artifacts.

To overcome the unwanted over enhancement and noise amplifying, the fuzzy logic-based histogram equalization technique is proposed for both gray scale and color images.
The proposed FHE method does not only preserve image brightness but also improves the local contrast of the original image. First, fuzzy histogram is computed using fuzzy set theory. Second, the fuzzy histogram is separated into two based on the median value of the original image. Finally, the $\mathrm{HE}$ approach is applied independently on each subhistogram to improve the contrast.

The remainder of this paper is organized as follows. The conventional histogram equalization is discussed in Section 2, and Section 3 presents the fuzzy image enhancement. The proposed algorithm for fuzzification and enhancement are presented in Sections 4 and 5. Simulation of the test images and the qualitative and quantitative comparison of the results are discussed in Sections 6 and 7. Finally, Section 8 concludes this paper.

\section{Histogram Equalization}

Consider a digital image, $f=\{f(i, j)\}$, which has the total number of $N$ pixels with gray levels in the range $[0, L-1]$. For a given image $f$, the probability density function $P\left(f_{k}\right)$ is defined as

$$
P\left(f_{k}\right)=\frac{n^{k}}{n}
$$

for $k=0,1,2, \ldots, L-1$, where $n^{k}$ represents the number of times that the gray level $f_{k}$ appears in the input image $f$ and $n$ is the total number samples in the input image. Note that $P\left(f_{k}\right)$ is associated with the histogram of input image which represents the number of pixels that have a specific intensity $f_{k}$. In fact, a plot of $f_{k}$ versus $n_{k}$ is called histogram of input image $f$. The respective cumulative density function is then defined as

$$
C\left(f_{k}\right)=\sum_{j=0}^{L-1} p\left(f_{j}\right)
$$

for $k=0,1,2, \ldots, L-1$. Note that $C\left(f_{L-1}\right)=1$ by definition. Histogram equalization is a method that maps the input image into entire dynamic range $\left(f_{0}, f_{L-1}\right)$ by using the cumulative distribution function as a transform function. That is, let us define a transform function $T(f)$ based on cumulative density function as

$$
T(f)=\left\{f_{0}+\left(f_{L-1}-f_{0}\right) C\left(f_{k}\right)\right\} .
$$

Then, the enhanced image of $\operatorname{HE} g=g(i, j)$ can be expressed as

$$
g(i, j)=T(f)=\{T(f(i, j)) \mid \forall f(i, j) \in f\},
$$

where $f$ and $g$ are the original and enhanced images, $(i, j)$ are the $2 \mathrm{D}$ coordinates of the images, and $T$ is the intensity transformation function, which maps the original image into the entire dynamic range $\left(f_{0}, f_{L-1}\right)$. However, HE produces an undesirable checkerboard effects on enhanced images. Another problem of this method is that it also enhances the noises in the input image along with the image features [1119]. In order to overcome these problems, several methods 
were proposed for certain purposes. For example, BBHE is proposed for preserving the mean brightness of a given image while enhancing the contrast. The concept will be introduced in the remainder of the section.

Let $f_{m}$ be the mean of the image $f$, and assume that $f_{m} \in$ $\{0, L-1\}$. Based on input mean $f_{m}$, the image is decomposed into two subimages $f_{L}$ and $f_{U}$ as

$$
f=f_{L} \cup f_{U}
$$

where

$$
\begin{aligned}
& f_{L}=\left\{f(i, j) \mid f(i, j) \leq f_{m}, \forall f(i, j) \in f\right\}, \\
& f_{U}=\left\{f(i, j) \mid f(i, j)>f_{m}, \forall f(i, j) \in f\right\} .
\end{aligned}
$$

Note that subimage $f_{L}$ is composed of $\left\{f_{0}, f_{1}, \ldots, f_{m}\right\}$ and the subimage $f_{U}$ is composed of $\left\{f_{m+1}, f_{m+2}, \ldots, f_{L-1}\right\}$.

Next, define the respective probability density functions of the subhistograms $f_{L}$ and $f_{U}$ as

$$
P_{L}\left(f_{k}\right)=\frac{n_{L}^{k}}{n_{L}},
$$

where $k=0,1, \ldots, m$, and

$$
P_{U}\left(f_{k}\right)=\frac{n^{k}}{n_{U}}
$$

where $k=m+1, m+2, \ldots, L-1$, in which $n_{U}^{k}$ and $n^{k}{ }_{L}$ represent the respective numbers of $f_{k}$ in $f_{L}$ and $f_{U}$, and $n_{L}$ and $n_{U}$ are the total number of samples in $f_{L}$ and $f_{U}$, respectively. Note that $P_{L}\left(f_{k}\right)$ and $P_{U}\left(f_{k}\right)$ are associated with the histogram of the input image which represents the number of pixels that have a specific intensity $f_{k}$. The respective cumulative density functions for subhistograms $f_{L}$ and $f_{U}$ are then defined as

$$
\begin{gathered}
C_{L}\left(f_{k}\right)=\sum_{j=0}^{m} p_{L}\left(f_{j}\right), \\
C_{U}\left(f_{k}\right)=\sum_{j=m+1}^{L-1} p_{U}\left(f_{j}\right) .
\end{gathered}
$$

Let us define the following transform functions based on cumulative density functions as

$$
\begin{gathered}
T_{L}\left(f_{k}\right)=f_{0}+\left(f_{m}-f_{0}\right) C_{L}\left(f_{k}\right), \\
T_{U}\left(f_{k}\right)=f_{m+1}+\left(f_{L-1}-f_{m+1}\right) C_{U}\left(f_{k}\right) .
\end{gathered}
$$

Based on these transform functions, the decomposed subimages are equalized independently and the composition of the resulting equalized sub-images which constitute the output image. The overall mapping function is defined as:

$$
\begin{aligned}
g(i, j) & =T_{L}\left(f_{k}\right) \cup T_{U}\left(f_{k}\right) \\
& = \begin{cases}f_{0}+\left(f_{m}-f_{0}\right) C_{L}\left(f_{k}\right) & \text { if } f \leq f_{m} \\
f_{m+1}+\left(f_{L-1}-f_{m+1}\right) C_{U}\left(f_{k}\right) & \text { else. }\end{cases}
\end{aligned}
$$

If we note that $0 \leq C_{L}\left(f_{k}\right), C_{U}\left(f_{k}\right) \leq 1$, it is easy to see that $T_{L}\left(f_{k}\right)$ equalizes the sub-image $f_{L}$ over the range $\left(f_{0}, f_{m}\right)$, whereas $T_{U}\left(f_{k}\right)$ equalizes the sub-image $f_{U}$ over the range $\left(f_{m+1}, f_{L-1}\right)$. As a consequence, the input image $f$ is equalized over the entire dynamic range $\left(f_{0}, f_{L-1}\right)$ with the constraint that the samples less than the input mean are mapped to $\left(f_{0}, f_{m}\right)$, and the samples greater than the mean are mapped to $\left(f_{m+1}, f_{L-1}\right)$.

\section{Fuzzy Image Enhancement}

Fuzzy image enhancement is done by mapping image gray level intensities into a fuzzy plane using membership functions, the membership functions are modified for contrast enhancement, and the fuzzy plane is mapped back to image gray level intensities. The aim is to generate an image of higher contrast than the original image by giving the larger weight to the gray levels that are closer to the mean gray level of the image than to those that are farther from the mean. In recent years, many researchers have applied fuzzy set theory to develop new techniques for image enhancement. Fuzzy set theory offers a mathematical framework for a new image understanding. An image of size $M \times N$ pixels and L gray levels can be considered as an array of fuzzy singletons, each having a value of membership denoting its degree of brightness relative to some brightness levels with $g=(0,1,2 \ldots L-1)[20]$. The fuzzy matrix corresponding to this image can be written as

$$
F=\bigcup_{m=1}^{M} \bigcup_{n=1}^{N} \frac{\mu_{m n}}{g_{m n}} \quad \text { with } \mu_{m n} \subseteq[0,1] \text {, }
$$

where $g_{m n}$ is the intensity of $(m, n)$ th pixel and $\mu_{m n}$ is its membership value. Fuzzy image processing consists of three stages: fuzzification (image coding), operations in the membership plane, and defuzzification (decoding of results). Fuzzification does mean that we assign image with one or more membership values regarding interesting properties (e.g. brightness, edginess, and homogeneity). After transformation of image into the membership plane (fuzzification), a suitable fuzzy approach modifies the membership values [21]. To achieve modified gray levels, the output of membership plane should be decoded (defuzzification). It means that the membership values are retransformed into the gray-level plane. For instance, to manage the grayness ambiguity, the gray levels must be fuzzified in relation to the location of image histogram. It means that each gray level is assigned to a degree of membership depending on its location in the histogram. Generally, dark pixels are assigned as low membership values, and bright pixels are assigned as high membership values.

3.1. Fuzzy Contrast/Brightness Adaptation. Contrast and brightness are image qualities that are specially important if the preprocessing is done for human perception [22]. There are two different ways to adapt the image contrast or brightness: gray level modification with suitable function $f$ :

$$
\bar{g}=f(g)
$$


or histogram operations. The performance of the first approach depends on the shape of the selected function. The second approach modifies the image histogram. The histogram equalization is one of the best known histogram techniques. The modified gray levels $\bar{g}$ are computed by

$$
\bar{g}=f_{h}(g)=(L-1) \sum_{i=0}^{g} \frac{h(i)}{M N},
$$

where the right side should be round off in order to achieve integer values for $\bar{g}$. Fuzzy techniques use different tools of fuzzy set to adjust image contrast: minimization of fuzziness, equalization using fuzzy expected value, hyperbolization, $\lambda$ enhancement, and enhancement using fuzzy relations.

\section{Proposed Work}

In the conventional histogram equalization method, the remapping of the histogram peaks (local maxima) takes place which leads to the introduction of undesirable artifacts and large change in mean image brightness. Hence, the proposed fuzzy logic-based histogram equalization is not only preserves the image brightness but also improves the local contrast of the original image. First, fuzzy histogram is created using fuzzy logic to handle the inexactness of gray level values in a better way, and it is separated into two subhistograms based on the median value of the original image. Then, each histogram is assigned to a new dynamic range. Finally, the HE approach is applied independently on each sub-histogram. The FHE technique consists of the following operational stages:

(i) image fuzzification and intensification;

(ii) fuzzy histogram computation;

(iii) histogram partitioning and equalization;

(iv) image defuzzification.

The following subsections include the details of the steps involved.

4.1. Image Fuzzification and Intensification. In image fuzzification, the gray level intensities are transformed to fuzzy plane whose value ranges between 0 and 1 . An image $f$ of size $M * N$ and intensity level in the range $(0, L-1)$ can be considered as a set of fuzzy singletons in the fuzzy set notation, each with a membership function denoting the degree of having some gray level. The fuzzy matrix $F$ corresponding to this image can be expressed as

$$
F=\left[\begin{array}{cccc}
\frac{\mu_{11}}{f_{11}} & \frac{\mu_{12}}{f_{12}} & \ldots & \frac{\mu_{1 N}}{f_{1 N}} \\
\frac{\mu_{21}}{f_{21}} & \frac{\mu_{22}}{f_{22}} & \ldots & \frac{\mu_{2 N}}{f_{2 N}} \\
\cdots & \cdots & & \ldots \\
\frac{\mu_{M 1}}{f_{M 1}} & \frac{\mu_{M 2}}{f_{M 2}} & \ldots & \frac{\mu_{M N}}{f_{M N}}
\end{array}\right] .
$$

Here $\mu_{i j}=0$ indicates dark, and $\mu_{i j}=1$ indicates bright. Any intermediate value refers to the grade of maximum gray level of the pixel. A set consisting of all $\mu_{i j}$ is called the fuzzy property plane of the image. In order to reduce the amount of image fuzziness, contrast intensification is applied to the fuzzy set $F$ to generate another fuzzy set, and the membership function of which is expressed as

$$
\mu_{F(i, j)}= \begin{cases}2 *\left(\mu_{i j}\right)^{2} & 0 \leq \mu_{i j} \leq 0.5 \\ 1-\left(2 *\left(1-\mu_{i j}\right)^{2}\right) & 0.5<\mu_{i j} \leq 1 .\end{cases}
$$

4.2. Fuzzy Histogram Computation. To enhance the image, we concentrate on contrast enhancement. This is achieved by making dark pixel more darker and making bright pixel brighter. Hence, fuzzy histogram is computed using (16). A fuzzy histogram is a sequence of real numbers $h(i), i \in$ $(0,1 \ldots, L-1)$, here $h(i)$ is the frequency of occurrence of gray levels that are around $i$. By considering the gray value $f(i, j)$ as a fuzzy number $\mu_{F(i, j)}$, the fuzzy histogram is computed as

$$
F \longleftarrow h(i)+\sum_{i} \sum_{j} \mu_{F(i, j)},
$$

where $\mu_{F(i, j)}$ is the fuzzy membership function. Fuzzy statistics is able to handle the inexactness of gray values in a much better way compared to classical crisp histograms thus producing a smooth histogram.

4.3. Histogram Partition and Equalization. Based on input median $M$, the fuzzy histogram $F$ is decomposed into two subhistograms $F_{L}$ and $F_{U}$ as

$$
F=F_{L} \cup F_{U},
$$

where

$$
\begin{aligned}
& F_{L}=\{F(i, j) \mid F(i, j) \leq M, \forall F(i, j) \in F\}, \\
& F_{U}=\{F(i, j) \mid F(i, j)>M, \forall F(i, j) \in F\} .
\end{aligned}
$$

Next, define the respective probability density functions of the sub histograms $F_{L}$ and $F_{U}$ as

$$
P_{L}\left(F_{k}\right)=\frac{n^{k}}{n_{L}},
$$

where $k=0,1, \ldots, m$, and

$$
P_{U}\left(F_{k}\right)=\frac{n_{U}^{k}}{n_{U}}
$$

where $k=m+1, m+2, \ldots, L-1$, in which $n_{U}^{k}$ and $n^{k}$ represent the respective numbers of $F_{k}$ in $F_{L}$ and $F_{U}$, and $n_{L}$ and $n_{U}$ are the total number of samples in $F_{L}$ and $F_{U}$, respectively. Note that $P_{L}\left(F_{k}\right)$ and $P_{U}\left(F_{k}\right)$ are associated with the fuzzy histogram of the input image which represents the number of pixels that have a specific intensity $F_{k}$. The respective cumulative density functions for sub-histograms $F_{L}$ and $F_{U}$ are then defined as

$$
\begin{gathered}
C_{L}\left(F_{k}\right)=\sum_{j=0}^{m} p_{L}\left(F_{j}\right), \\
C_{U}\left(F_{k}\right)=\sum_{j=m+1}^{L-1} p_{U}\left(F_{j}\right) .
\end{gathered}
$$


Let us define the following transform functions based on cumulative density functions as

$$
\begin{gathered}
T_{L}\left(F_{k}\right)=F_{0}+\left(M-F_{0}\right) C_{L}\left(F_{k}\right), \\
T_{U}\left(F_{k}\right)=M+1+\left(F_{L-1}-M+1\right) C_{U}\left(F_{k}\right) .
\end{gathered}
$$

Based on these transform functions, the decomposed sub-images are equalized independently, and the composition of the resulting equalized sub-images which constitute the output image. The output image $g=\{g(i, j)\}$ is expressed as

$$
g(i, j)=T_{L}\left(F_{L}\right) \cup T_{U}\left(F_{U}\right)
$$

where

$$
\begin{aligned}
T_{L}\left(F_{L}\right) & =\left\{T_{L}(F(i, j)) \mid \forall F(i, j) \in F_{L}\right\}, \\
T_{U}\left(F_{U}\right) & =\left\{T_{U}(F(i, j)) \mid \forall F(i, j) \in F_{U}\right\} .
\end{aligned}
$$

If we note that $0 \leq C_{L}\left(F_{k}\right), C_{U}\left(F_{k}\right) \leq 1$, it is easy to see that $T_{L}\left(F_{L}\right)$ equalizes the sub-image $F_{L}$ over the range $\left(F_{0}, M\right)$, whereas $T_{U}\left(F_{U}\right)$ equalizes the sub-image $F_{U}$ over the range $\left(M+1, F_{L-1}\right)$. As a consequence, the input image $\mathrm{F}$ is equalized over the entire dynamic range $\left(F_{0}, F_{L-1}\right)$ with the constraint that the samples less than the input median are mapped to $\left(F_{0}, M\right)$ and the samples greater than the median are mapped to $\left(M+1, F_{L-1}\right)$.

4.4. Image Defuzzification. Image defuzzification is the inverse of fuzzification. The algorithm maps the fuzzy plane back to gray level intensities. Finally, the enhanced image $G(i, j)$ can be obtained by the following inverse transformation:

$$
G(i, j)=T^{-1}(g(i, j))=\bigcup_{i=1}^{M} \bigcup_{j=1}^{N} g(i, j) *(L-1),
$$

where $G(i, j)$ denotes the gray level of the $(i, j)$ th pixel in the enhanced image and $T^{-1}$ denotes the inverse transformation of $T$. This brightness preserving procedure ensures that the mean intensity of the image obtained after process is the same as that of the input.

\section{Contrast Enhancement of Color Images}

Most electronic equipments acquire and display the color images. In this respect, the method of enhancing color images would be of better interest. Hence, the contrast enhancement can be easily extended to color images. The most obvious way to extend the proposed gray-scale contrast enhancement to color images is to apply the method to luminance component only and to preserve the chrominance components. This method produces better perceptible results as compared to other conventional methods.

\section{Image Quality Assessment}

Image quality assessment (IQA) aims to use computational models to measure the image quality consistently with subjective evaluations. IQA indices used in our evaluation include entropy or average information contents (AIC) and natural image quality evaluator (NIQE) index for measuring image quality.

The AIC is used to measure the content of an image, where a higher value indicates an image with richer details. Higher value of the AIC indicates that more information is brought out from the images. NIQE is used for measuring image quality. Smaller NIQE indicates the better image quality. The average information contents or entropy is defined as

$$
\mathrm{AIC}=-\sum_{k=0}^{L-1} P(k) \log P(k),
$$

where $P(k)$ is the probability density function of the $k$ th gray level [23]. Moreover, natural image quality evaluator (NIQE) index is used for measuring image quality [24, 25]. It is mainly based on the construction of a "quality aware" collection of statistical features based on a simple and successful space domain natural scene statistic (NSS) model. These features are derived from a corpus of natural, undistorted images. The quality of a given test image is then expressed as the distance between a multivariate gaussian (MVG) fit of the NSS features extracted from the test image and a MVG model of the quality aware features extracted from the corpus of natural images. The NIQE score typically has a value between 0 and 100 (0 represents the best quality, 100 the worst).

\section{Results and Discussion}

In this section, comparison between the proposed method and several other conventional methods such as histogram equalization (HE), brightness preserving bi-histogram equalization (BBHE), minimum mean brightness error bihistogram equalization (MMBEBHE), and brightness preserving dynamic fuzzy histogram equalization (BPDFHE) are presented. A subjective assessment to compare the visual quality of the images is carried out. An Average information contents (AIC) and natural image quality evaluator (NIQE) index is used to assess the effectiveness of the proposed method. The proposed method was tested with several gray scale and color images and had been compared with other conventional methods $\mathrm{HE}$, BBHE, MMBEBHE, and BPDFHE. The test images used for the experiments are available on the website http:// www.ponomarenko.info/tid2008.htm.

Figure 1 shows the resulting images obtained by the various existing methods and proposed method for the gray scale image. The histograms of all resultant images in Figure 1 are illustrated in Figure 2. Figure 1(b) shows that HE provides a significant improvement in image contrast. However, it also amplifies the noise level of the images along with some artifacts and undesirable side effects such as washed-out appearance. Figure 1(c) shows that the BBHE method produces unnatural look and insignificant enhancement to the resultant image. However, it also has unnatural look because of over enhancement in brightness. This can be proved from the result shown in the histogram of two methods shown in Figures 2(b) and 2(c) which are squeezed to left tail of the histogram. 


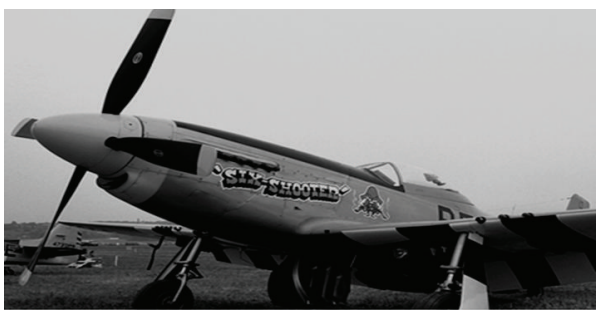

(a)

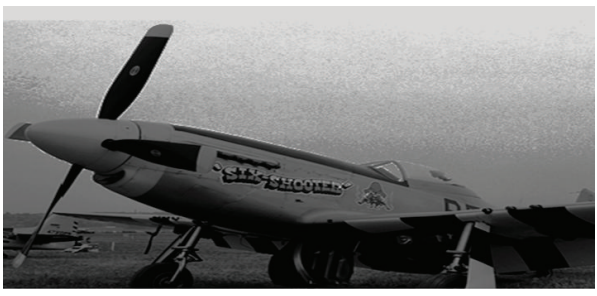

(c)

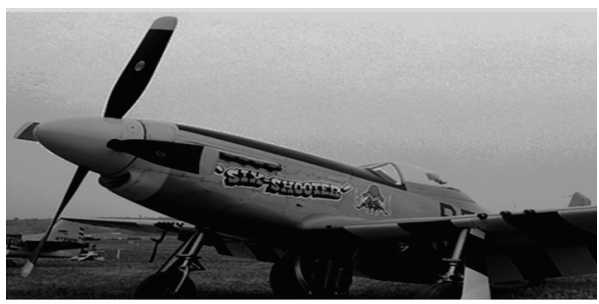

(e)

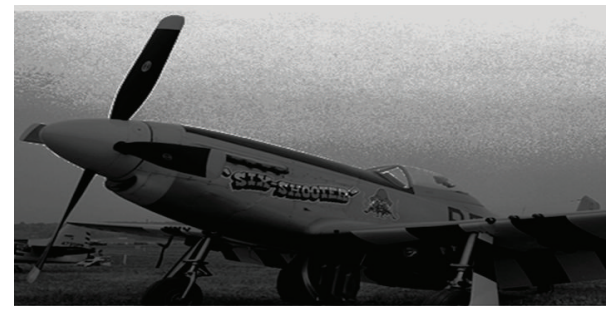

(b)

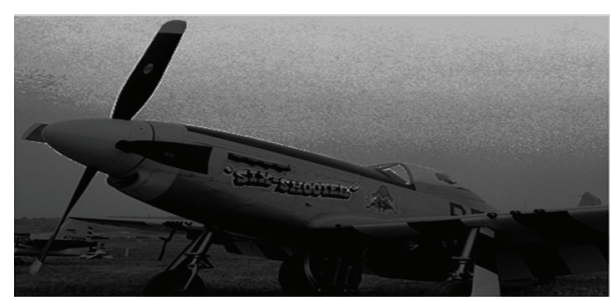

(d)

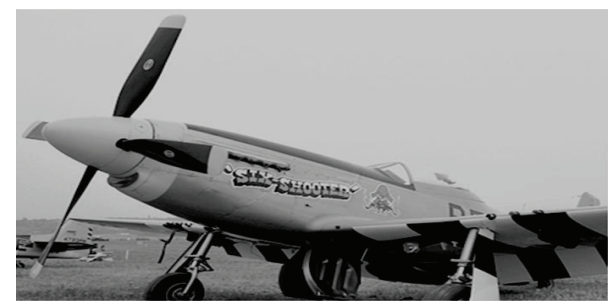

(f)

FIGURE 1: Simulation results of the gray scale image (Image Id: I20). (a) Original image, (b) HE-ed image, (c) BBHE-ed image, (d) MMBEBHEed image, (e) BPDFHE-ed image, and (f) proposed method.

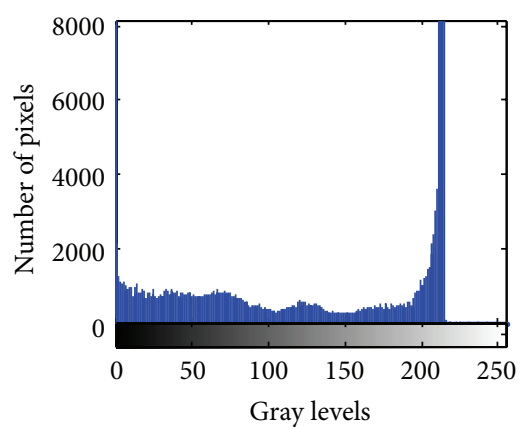

(a)

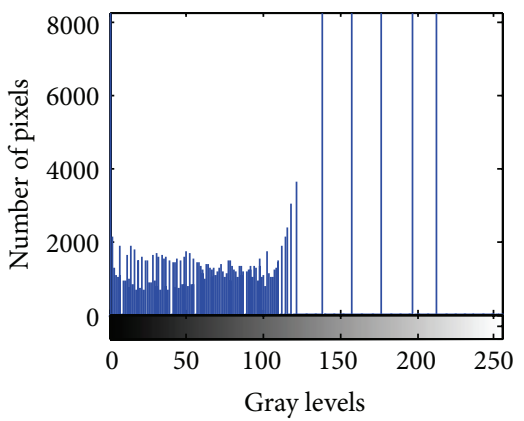

(d)

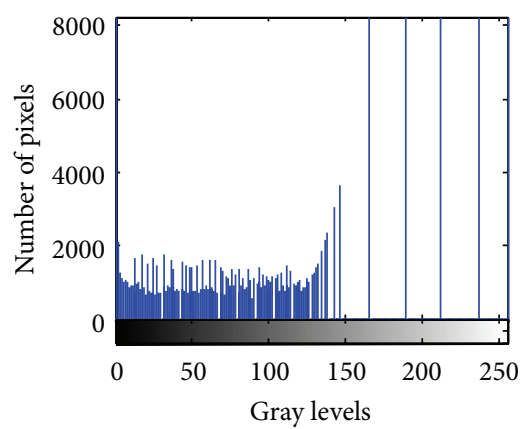

(b)

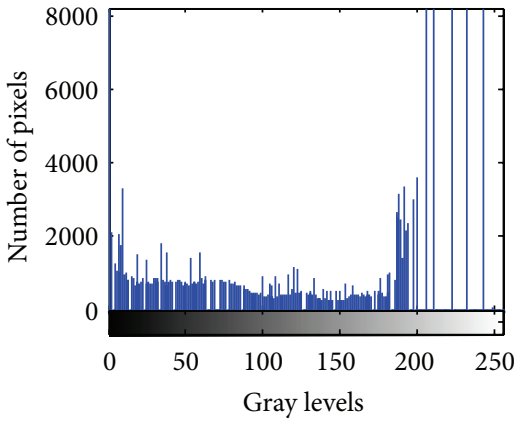

(e)

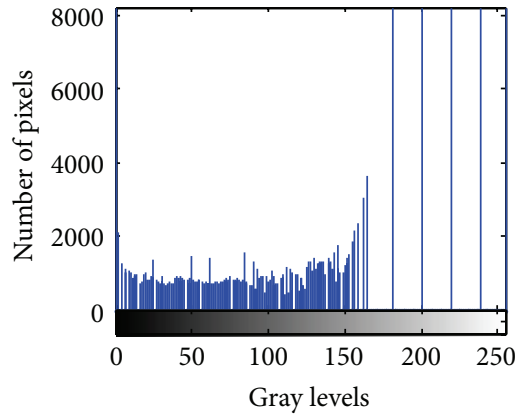

(c)

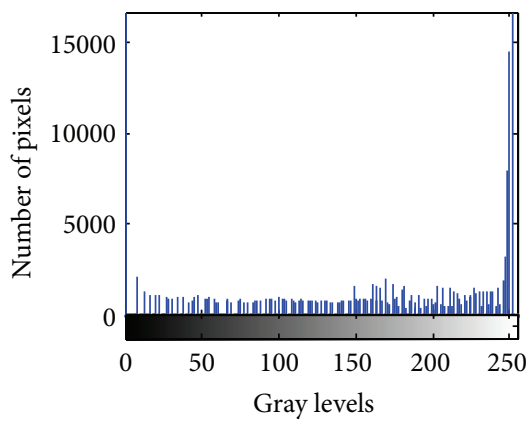

(f)

FIGURE 2: Histogram of simulated gray scale image (Image Id: I20). (a) Original histogram, (b) HE-ed histogram, (c) BBHE-ed histogram, (d) MMBEBHE-ed histogram, (e) BPDFHE-ed histogram, and (f) proposed histogram. 


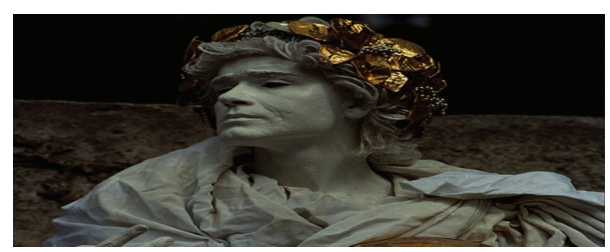

(a)

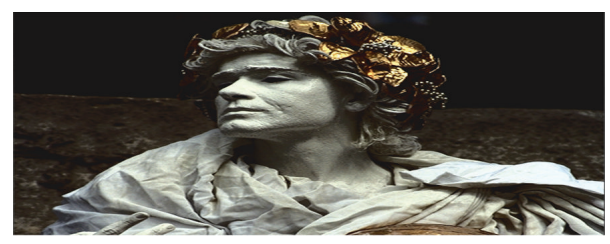

(c)

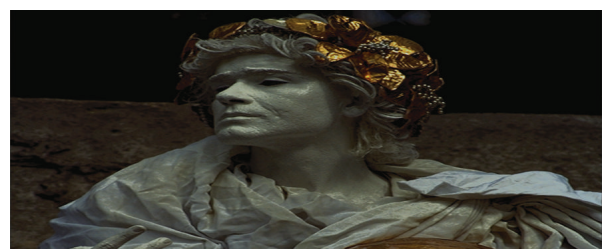

(e)

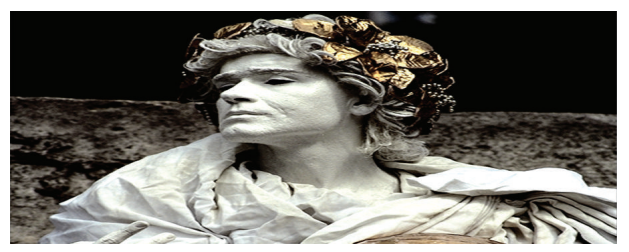

(b)

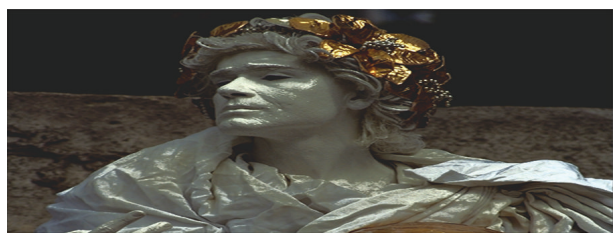

(d)

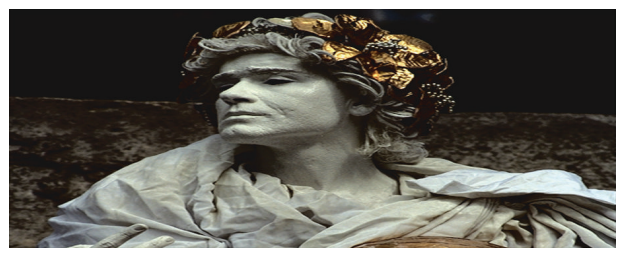

(f)

FIGURE 3: Simulation results of the color image (Image Id: I17). (a) Original image, (b) HE-ed image, (c) BBHE-ed image, (d) MMBEBHE-ed image, (e) BPDFHE-ed image, and (f) Proposed Method.

The results of MMBEBHE and BPDFHE (Figures 1(d) and 1(e)) show good contrast enhancement, and they also cause more annoying side effects depending on the variation of gray level distribution in the histogram. The output histograms of MMBEBHE and BPDFHE fail to achieve smooth distribution between low and high gray levels. Thus, the enhancement results of MMBEBHE and BPDFHE are visually unpleasing. The results of HE, BBHE, MMBEBHE, and BPDFHE show that they do not prevent the washed-out appearance in overall image due to significant change in brightness. The result shows that the proposed method preserves the naturalness of image and also prevents the side effect due to the significant change in brightness effectively. By visually inspecting the images on these figures, we can clearly see that only the proposed method is able to generate natural looking image and still offer contrast enhancement. Since the output histogram of proposed method achieves a smoother distribution between low and high gray values, as shown in Figure 2(f), its result is more natural looking. The histogram of FHE is successfully distributed evenly to its full dynamic range as compared to other conventional methods. Figure 3 shows the resulting images obtained by the various existing methods such as HE, BBHE, MMBEBHE, BPDFHE, and proposed method for the color image. The performance of FHE is evaluated on various color images. These images are shown in Figure 4. Figures 5 and 6 show the comparison of average AIC and NIQE values between the proposed method and other conventional methods for various images. Further, the qualities of the test images which are enhanced using the aforementioned techniques that are measured in terms of
AIC and NIQE are given in Tables 1 and 2. According to Table 1, the FHE produces the highest AIC and thus becomes the best method to bring out the details of the images. Table 2 shows the natural image quality evaluator index of tested images. From Table 2, it is found that FHE produces lowest NIQE values as compared to the other conventional methods. Hence, FHE produces enhanced images with natural looking. Based upon qualitative and quantitative analyses, the proposed FHE method has been found effective in enhancing contrasts of images in comparison to a few existing methods. In addition, fuzzy histogram equalized images uses full dynamic range of the pixel values for maximum contrast. The performance of the proposed method has been compared with five state-of-the-art methods, both quantitatively and visually. Experimental result shows that the proposed method not only outperforms in contrast enhancement but also provides good visual representation in visual comparison. Hence, FHE gives better visual quality images.

\section{Conclusion}

In this work, Fuzzy logic-based histogram equalization is presented for image contrast enhancement. FHE uses fuzzy statistics of digital images to handle the inexactness of gray level values in a better way compared to classical crisp histograms, resulting in improved performance. It can also improve the color content, brightness, and contrast of an image automatically. This algorithm was tested on different gray scale and color images (they were extracted 

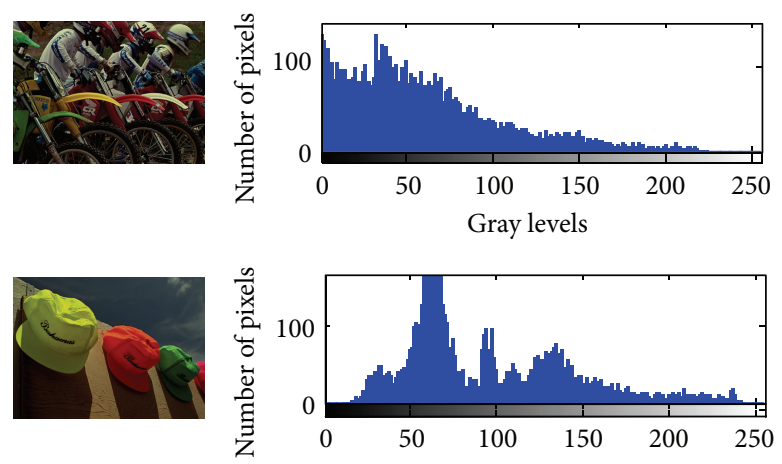

Gray levels
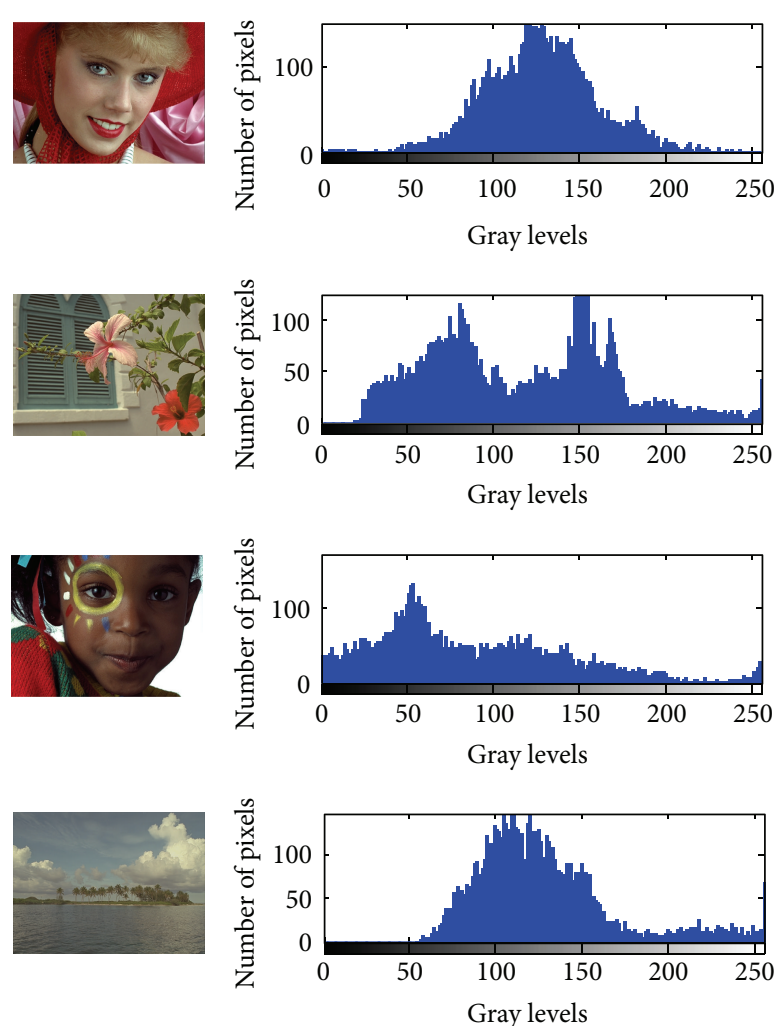

(a)
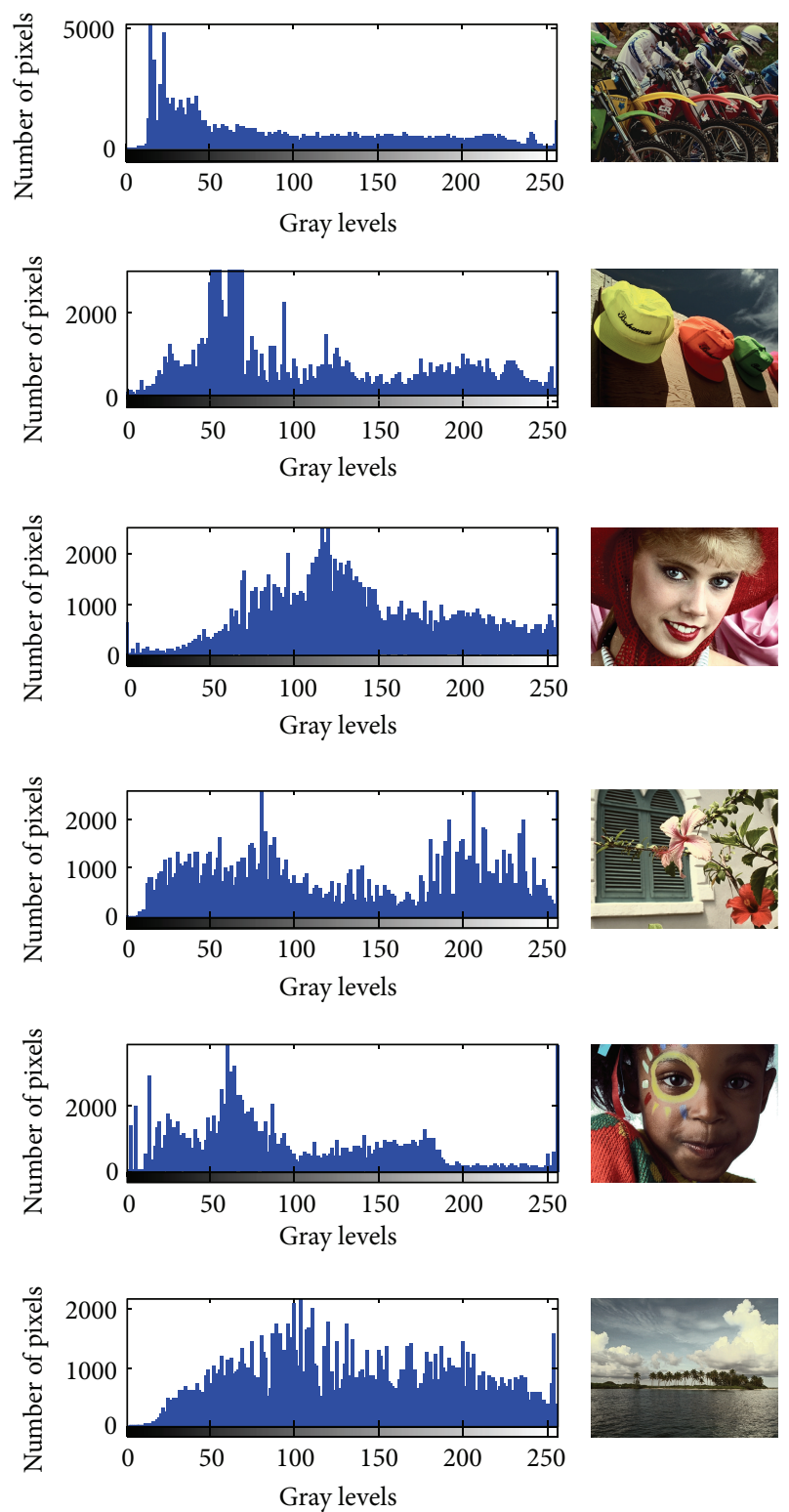

(b)

FIgURE 4: Contrast enhancement results on the tested images: (a) Original image with its histogram, (b) proposed FHE-ed image with its histogram.

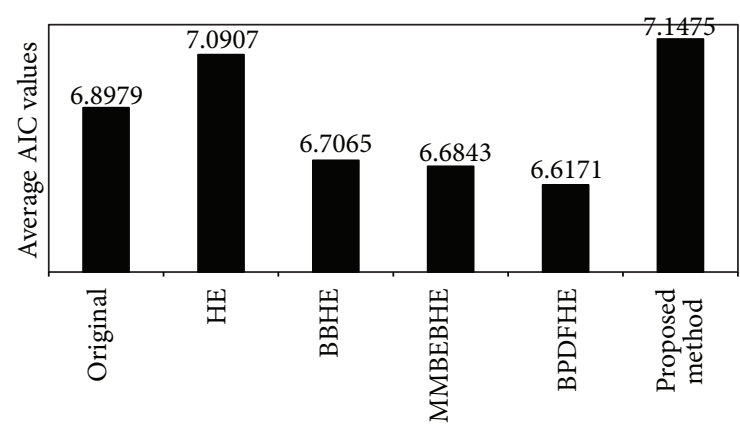

FIGURE 5: Comparison of average AIC values.

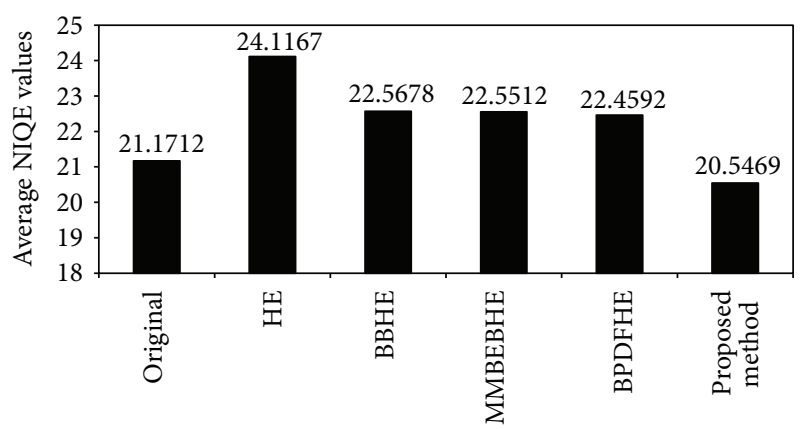

Figure 6: Comparison of average NIQE values. 
TABLE 1: Comparison of AIC values.

\begin{tabular}{|c|c|c|c|c|c|c|}
\hline Image ID & Original & H.E & BBHE & MMBEBHE & BPDFHE & Proposed method \\
\hline $\mathrm{I} 03$ & 7.1366 & 7.3239 & 6.9263 & 6.9541 & 6.9032 & 7.3794 \\
\hline I04 & 7.0484 & 7.3835 & 6.8931 & 6.9294 & 6.7861 & 7.4470 \\
\hline I05 & 6.5355 & 6.8877 & 6.3495 & 6.3735 & 6.1581 & 7.0169 \\
\hline I06 & 7.4612 & 7.4275 & 7.3512 & 7.3435 & 7.1991 & 7.4481 \\
\hline I07 & 7.1888 & 7.4265 & 7.0187 & 7.0751 & 6.9260 & 7.3796 \\
\hline $\mathrm{I} 12$ & 7.1710 & 7.4952 & 6.9772 & 6.9376 & 6.8926 & 7.6427 \\
\hline I17 & 6.0779 & 6.1511 & 5.8237 & 5.7842 & 5.7094 & 6.2738 \\
\hline I19 & 7.5198 & 7.3572 & 7.3502 & 7.3543 & 7.3152 & 7.6406 \\
\hline $\mathrm{I} 20$ & 5.6379 & 5.9485 & 5.3846 & 5.2187 & 5.4697 & 5.6576 \\
\hline $\mathrm{I} 21$ & 7.2026 & 7.5061 & 6.9909 & 6.8722 & 6.8118 & 7.5895 \\
\hline Average & 6.8979 & 7.0907 & 6.7065 & 6.6843 & 6.6171 & 7.1475 \\
\hline
\end{tabular}

TABLE 2: Comparison of NIQE values.

\begin{tabular}{|c|c|c|c|c|c|c|}
\hline Image ID & Original & H.E & BBHE & MMBEBHE & BPDFHE & Proposed method \\
\hline I03 & 17.3988 & 23.3811 & 17.5405 & 18.8037 & 17.9547 & 17.3112 \\
\hline I04 & 19.5606 & 20.3620 & 18.4988 & 20.0583 & 21.1219 & 17.0081 \\
\hline I05 & 30.7510 & 30.4857 & 24.4350 & 29.5651 & 26.7359 & 25.4919 \\
\hline I06 & 19.6830 & 23.0644 & 20.3643 & 20.7971 & 21.7855 & 20.3254 \\
\hline I07 & 21.0681 & 20.9864 & 21.1715 & 22.1464 & 20.8622 & 21.3704 \\
\hline $\mathrm{I} 12$ & 18.9775 & 24.8047 & 24.9042 & 20.7823 & 24.8195 & 21.1546 \\
\hline I17 & 19.3532 & 22.0394 & 24.1477 & 22.9697 & 21.3077 & 18.8063 \\
\hline I19 & 22.5296 & 25.3067 & 24.0775 & 24.0815 & 23.6979 & 25.1392 \\
\hline $\mathrm{I} 20$ & 20.6246 & 23.5422 & 23.0723 & 20.3540 & 21.3669 & 18.2896 \\
\hline $\mathrm{I} 21$ & 21.7665 & 27.1946 & 27.4668 & 25.9539 & 24.9402 & 20.5731 \\
\hline Average & 21.1712 & 24.1167 & 22.5678 & 22.5512 & 22.4592 & 20.5469 \\
\hline
\end{tabular}

from the TID2008 database). The qualitative and subjective enhancement performance of the proposed FHE algorithm was evaluated and compared to the other conventional methods for different gray scale and color natural images. The performance of proposed FHE algorithm was evaluated and compared in terms of AIC and NIQE indices. The simulation results demonstrated that the proposed FHE algorithm provided better results as compared to conventional methods such as histogram equalization (HE), brightness preserving bi-histogram equalization (BBHE), minimum mean brightness error bi-histogram equalization (MMBEBHE), and brightness preserving dynamic fuzzy histogram equalization (BPDFHE) for different color images. The visual enhancement results of proposed FHE algorithm were also better as compared to the other conventional methods for different gray scale and color natural images. Observing from the simulation results obtained, the FHE has produced the best performance for both qualitative and quantitative evaluations. Experimental results demonstrate that the proposed method can effectively and significantly eliminate the washed-out appearance and adverse artifacts induced by several existing methods. This method is simple and suitable for consumer electronic products.

\section{References}

[1] X. Su, W. Fang, Q. Shen, and X. Hao, "An image enhancement method using the quantum-behaved particle swarm optimization with an adaptive strategy," Mathematical Problems in Engineering, vol. 2013, Article ID 824787, 13 pages, 2013.

[2] Y. Yang, J. Zhang, and X. Huang, "Adaptive image enhancement algorithm combining kernel regression and local homogeneity," Mathematical Problems in Engineering, vol. 2010, Article ID 693532, 14 pages, 2010.

[3] C. G. Ravichandran and V. Magudeeswaran, "An efficient method for contrast enhancement in still images using histogram modification framework," Journal of Computer Science, vol. 8, no. 5, pp. 775-779, 2012.

[4] Y. Kim, "Contrast enhancement using brightness preserving bi-histogram equalization," IEEE Transactions on Consumer Electronics, vol. 43, no. 1, pp. 1-8, 1997.

[5] Y. Wan, Q. Chen, and B.-M. Zhang, "Image enhancement based on equal area dualistic sub-image histogram equalization method," IEEE Transactions on Consumer Electronics, vol. 45, no. 1, pp. 68-75, 1999.

[6] S. D. Chen and A. R. Ramli, "Minimum mean brightness error bi-histogram equalization in contrast enhancement," IEEE Transactions on Consumer Electronics, vol. 49, no. 4, pp. 13101319, 2003. 
[7] S. D. Chen and A. R. Ramli, "Contrast enhancement using recursive mean-separate histogram equalization for scalable brightness preservation," IEEE Transactions on Consumer Electronics, vol. 49, no. 4, pp. 1301-1309, 2003.

[8] M. Abdullah-Al-Wadud, M. H. Kabir, M. A. A. Dewan, and O. Chae, "A dynamic histogram equalization for image contrast enhancement," IEEE Transactions on Consumer Electronics, vol. 53, no. 2, pp. 593-600, 2007.

[9] N. S. P. Kong and H. Ibrahim, "Color image enhancement using brightness preserving dynamic histogram equalization," IEEE Transactions on Consumer Electronics, vol. 54, no. 4, 2008.

[10] D. Sheet, H. Garud, A. Suveer, M. Mahadevappa, and J. Chatterjee, "Brightness preserving dynamic fuzzy histogram equalization," IEEE Transactions on Consumer Electronics, vol. 56, no. 4, pp. 2475-2480, 2010.

[11] H. Kabir, A. Al-Wadud, and O. Chae, "Brightness preserving image contrast enhancement using weighted mixture of global and local transformation functions," International Arab Journal of Information Technology, vol. 7, no. 4, pp. 403-410, 2010.

[12] S.-C. Huang and H.-Y. Chien, "Image contrast enhancement for preserving mean brightness without losing image features," Engineering Applications of Artificial Intelligence, vol. 6, pp. 1487-1492, 2013.

[13] H. Ibrahim and N. S. Pik Kong, "Image sharpening using subregions histogram equalization," IEEE Transactions on Consumer Electronics, vol. 55, no. 2, pp. 891-895, 2009.

[14] K. Gunaseelan and E. Seethalakshmi, "Image resolution and contrst enhancement using singular value and discrete wavelet decomposition," Journal of Scientific and Industrial Research, vol. 72, pp. 31-35, 2013.

[15] S. H. Lim, N. A. M. Isa, C. H. Ooi, and K. K. V. Toh, "A new histogram equalization method for digital image enhancement and brightness preservation," Signal, Image and Video Processing, 2013.

[16] A. M. Reza, "Realization of the contrast limited adaptive histogram equalization (CLAHE) for real-time image enhancement," Journal of VLSI Signal Processing Systems for Signal, Image, and Video Technology, vol. 38, no. 1, pp. 35-44, 2004.

[17] R. C. Gonzalez, R. E. Woods, and S. L. Eddins, Digital Image Processing, Prentice Hall, Upper Saddle River, NJ, USA, 2004.

[18] S. Chen and A. Beghdadi, "Natural enhancement of color image," Eurasip Journal on Image and Video Processing, vol. 2010, Article ID 175203, 30 pages, 2010.

[19] M. M. Riaz and Abdul Ghafoor, "Through-wall image enhancement based on singular value decomposition," International Journal of Antennas and Propagation, vol. 2012, Article ID 961829, 20 pages, 2012.

[20] K. Hasikin and N. A. M. Isa, "Adaptive fuzzy contrast factor enhancement technique for low contrast and non-uniform illumination images," Signal, Image and Video Processing, 2012.

[21] N. Y. Suple and S. M. Kharad, "Basic approach to image contrast enhancement with fuzzy inference system," International Journal of Scientific and Research Publications, vol. 3, no. 6, 2013.

[22] E. E. Kerre and M. Nachtegael, Eds., Fuzzy Techniques in Image Processing, Physica, Heidelberg, Germany, 2000.

[23] Y. Chang and C. Chang, "A simple histogram modification scheme for contrast enhancement," IEEE Transactions on Consumer Electronics, vol. 56, no. 2, pp. 737-742, 2010.

[24] A. K. Moorthy and A. C. Bovik, "Blind image quality assessment: from natural scene statistics to perceptual quality," IEEE Transactions on Image Processing, vol. 20, no. 12, pp. 3350-3364, 2011.
[25] A. Mittal, R. Soundararajan, and A. C. Bovik, "Making a completely blind image quality analyzer," IEEE Signal Processing Letters, vol. 20, no. 3, 2013. 


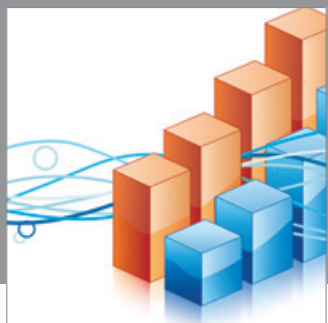

Advances in

Operations Research

mansans

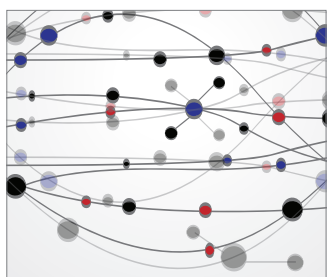

The Scientific World Journal
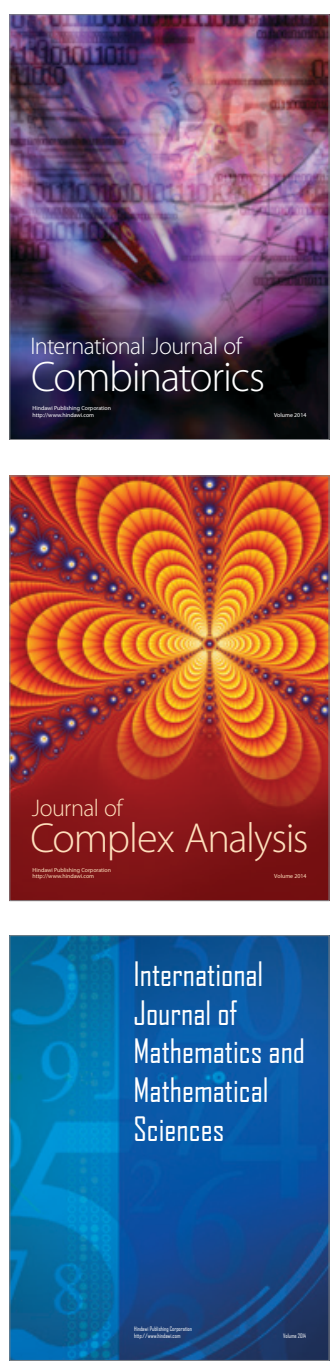
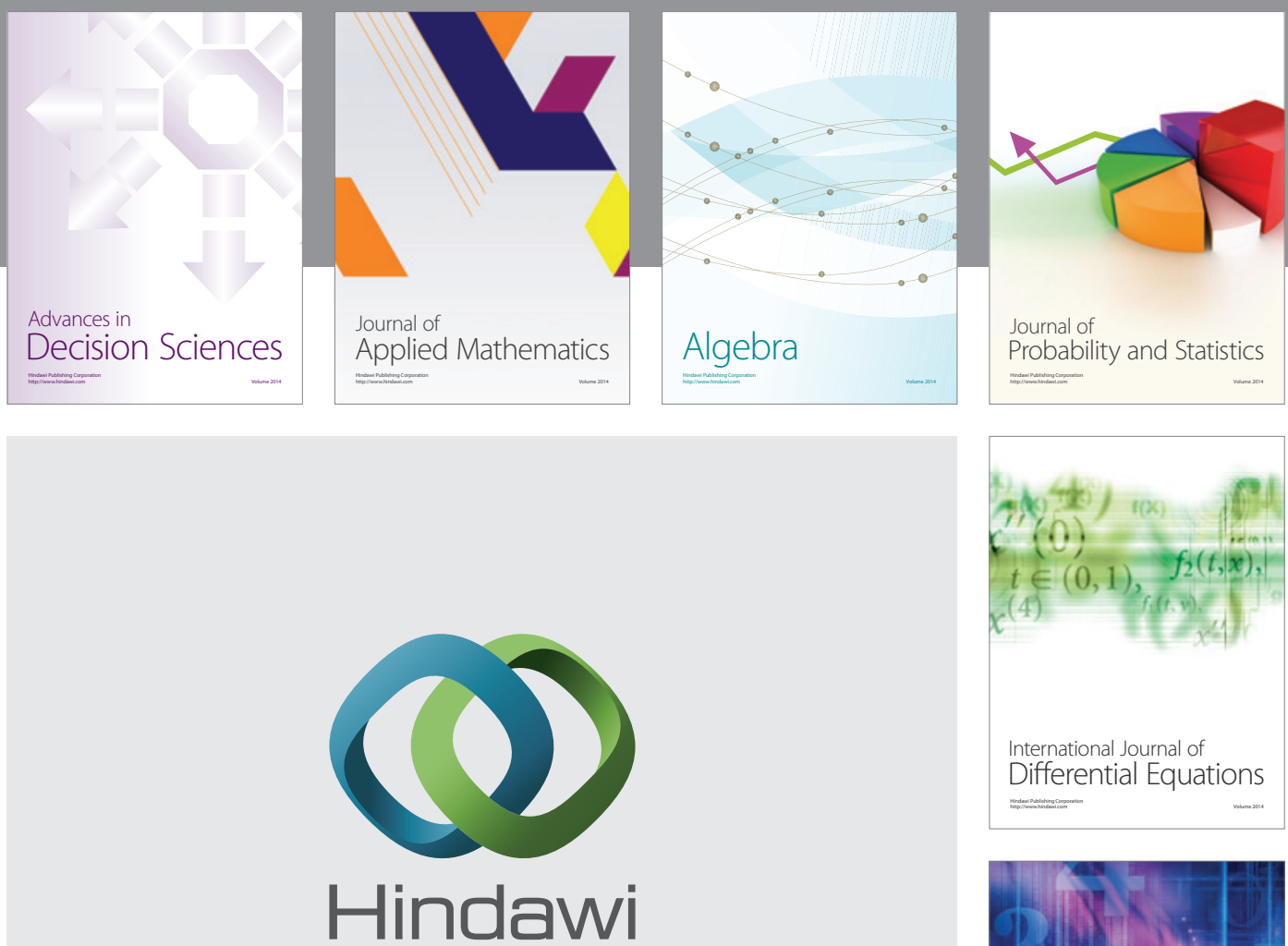

Submit your manuscripts at http://www.hindawi.com
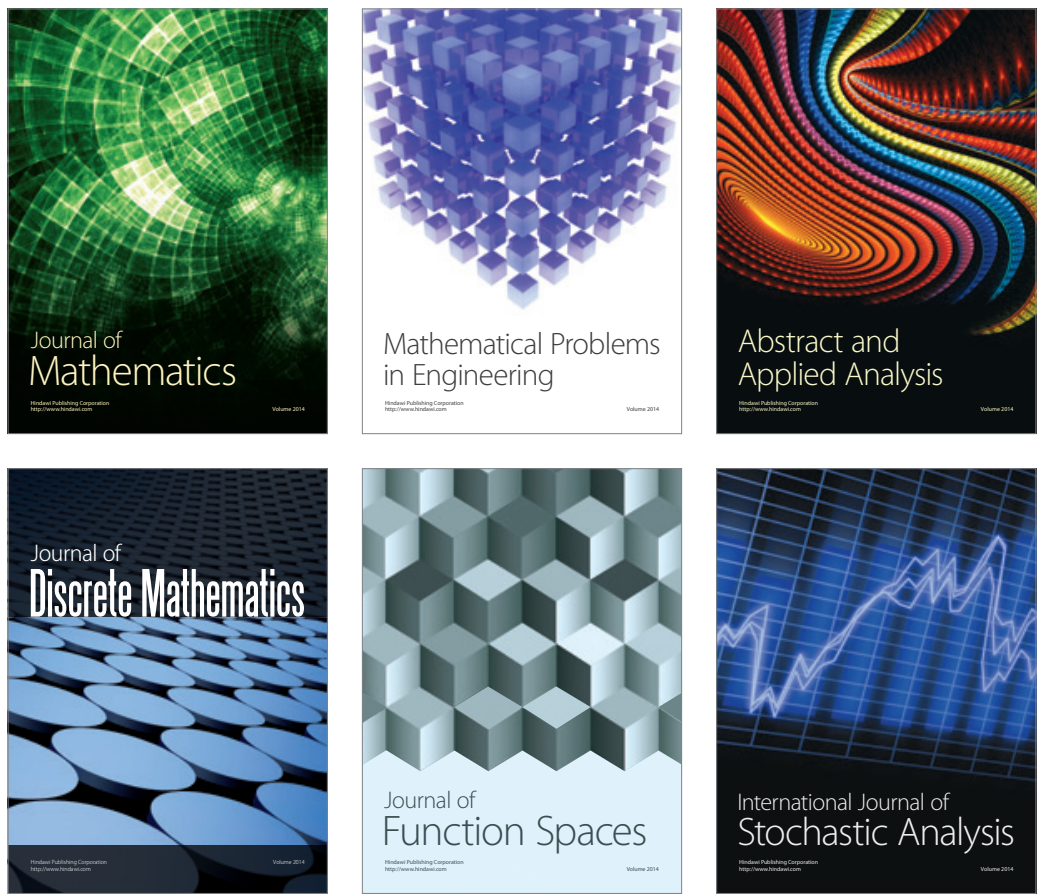

Journal of

Function Spaces

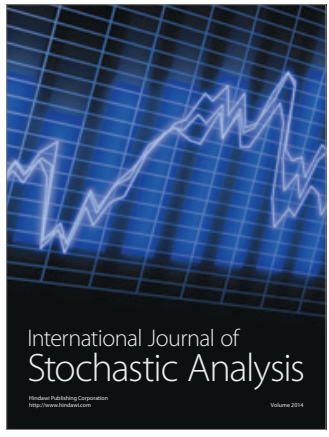

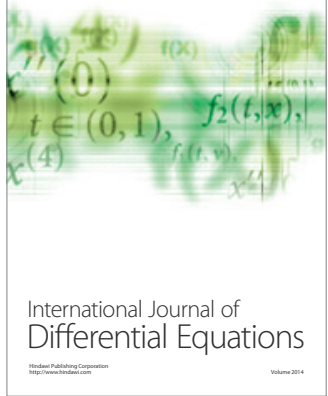
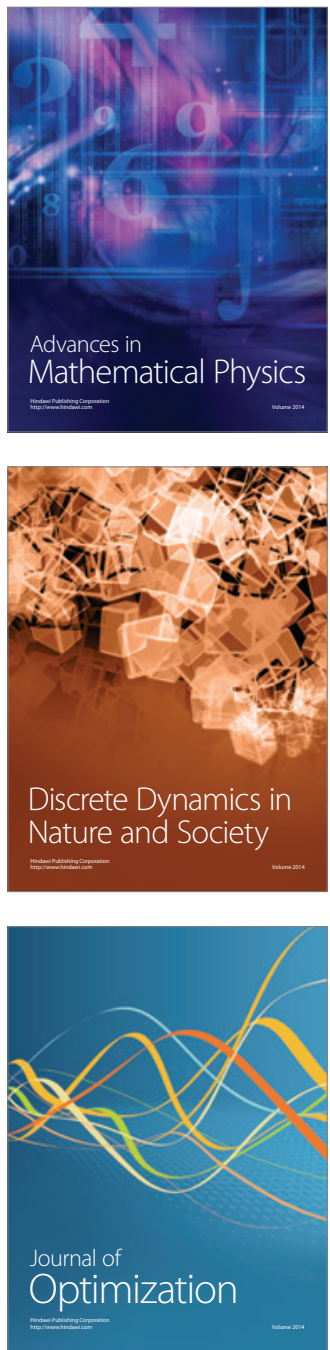